DESY 04-216

November 2004

\title{
Maximal Temperature in Flux Compactifications
}

\author{
Wilfried Buchmüller, Koichi Hamaguchi, Oleg Lebedev \\ Deutsches Elektronen-Synchrotron DESY, 22603 Hamburg, Germany \\ Michael Ratz \\ Physikalisches Institut der Universität Bonn, \\ Nussallee 12, 53115 Bonn, Germany
}

\begin{abstract}
Thermal corrections have an important effect on moduli stabilization leading to the existence of a maximal temperature, beyond which the compact dimensions decompactify. In this note, we discuss generality of our earlier analysis and apply it to the case of flux compactifications. The maximal temperature is again found to be controlled by the supersymmetry breaking scale, $T_{\text {crit }} \sim \sqrt{m_{3 / 2} M_{\mathrm{P}}}$.
\end{abstract}


In string theory gauge and Yukawa couplings are dynamical quantities whose values are determined by expectation values of scalar fields (moduli). In perturbation theory their potential is flat, and their stabilization is a topic of central importance in string theory. Recently, it has been proposed that a complete stabilization of all moduli fields [1] is possible using a combination of fluxes [2] and non-perturbative effects such as D-brane instantons and gaugino condensation [3].

The moduli potentials receive important thermal corrections $[4,5]$. These corrections destabilize the moduli at sufficiently high temperature, i.e. drive them to the 'run-away' minimum [6] where the compact dimensions decompactify. In order to avoid this cosmological disaster the temperature in the early universe has to be smaller than a maximal temperature $T_{\text {crit }}$.

In the following we discuss generality of our previous analysis [5] on dilaton destabilization by thermal effects and apply it to the KKLT scenario [1]. We find again that, generically, the maximal temperature is given by the scale of supersymmetry breaking, i.e., $T_{\text {crit }} \sim \sqrt{m_{3 / 2} M_{\mathrm{P}}}$. Temperature effects in some field theoretical flux compactifications have recently been considered in [7].

\section{Moduli destabilization at high temperature}

In thermal field theory, the free energy $F$ plays the role of an effective potential. For a Yang-Mills theory at high temperature $T$, the free energy has a perturbative expansion in terms of the gauge coupling $g$,

$$
F(g, T)=-\frac{\pi^{2} T^{4}}{24}\left\{\alpha_{0}+\alpha_{2} g^{2}+\mathcal{O}\left(g^{3}\right)\right\}
$$

The crucial point of our subsequent discussion is that $F$ increases with increasing $g$. For a supersymmetric $\mathrm{SU}\left(N_{c}\right)$ gauge theory with $N_{f}$ matter multiplets, one has (cf. [8]) $\alpha_{0}=N_{c}^{2}+2 N_{c} N_{f}-1$ and

$$
\alpha_{2}=-\frac{3}{8 \pi^{2}}\left(N_{c}^{2}-1\right)\left(N_{c}+3 N_{f}\right)<0 .
$$

To order $\mathcal{O}\left(g^{2}\right)$ the free energy is determined by one- and two-loop diagrams (cf. Fig. 1). The qualitative behaviour of the free energy, $\partial F / \partial g^{2}>0$, is also valid at higher-loop level and even non-perturbatively for $g=\mathcal{O}(1)$ [5]. Furthermore, it holds for Abelian theories and for Yukawa couplings.

In string theory the gauge coupling is related to some modulus $\Phi$,

$$
g^{2}=\frac{\kappa}{\Phi},
$$

where $\kappa$ is a constant. Since thermal effects increase the effective potential, they will drive the modulus towards larger values, corresponding to smaller couplings, and eventually destabilize the system. From Eq. (10) one obtains

$$
V_{\mathrm{th}}(\Phi, T) \equiv T^{4} v_{\mathrm{th}}(\Phi)=T^{4}\left(a_{0}+a_{2} \frac{1}{\Phi}+\ldots\right)
$$



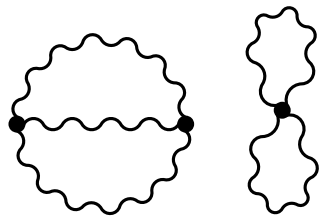

(a) Gauge-gauge.
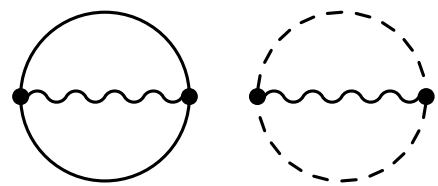

(b) Gauge-matter.

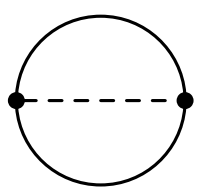

(c) Matter-matter.

Figure 1: Examples of two-loop diagrams contributing to the effective potential. Wavy lines represent gauge fields, while matter fields are indicated by solid and dashed lines.

where $a_{0}$ and $a_{2}$ are constants, with $a_{2}>0$. Clearly, the minimum of this potential is at $\Phi \rightarrow \infty$.

The effective potential for $\Phi$ is the sum of the zero-temperature potential $V$ and the thermal correction,

$$
V_{\text {eff }}(\Phi, T)=V_{\mathrm{th}}(\Phi, T)+V(\Phi)
$$

The supergravity potential $V(\Phi)$ for moduli is generated non-perturbatively. In known examples, it is related to supersymmetry breaking at least for some moduli. Such potentials allow one to stabilize the moduli at appropriate values, yet there is always the 'run-away' minimum at $\Phi \rightarrow \infty$, which is separated from the local minimum by a barrier related to SUSY breaking (Fig. 2).

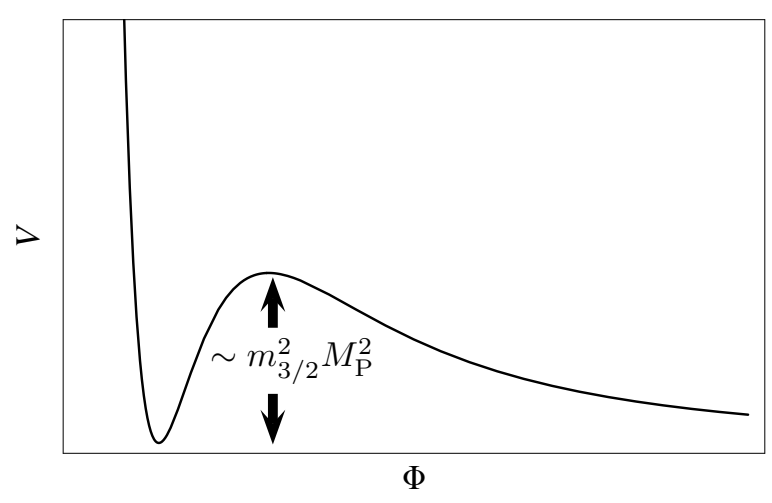

Figure 2: Typical supergravity potential for the modulus $\Phi$. The local minimum is separated from the 'run-away' one by a barrier related to SUSY breaking.

Clearly, when the temperature is high enough, the thermal potential (44) will dominate and drive $\Phi$ to infinity. Since the size of the potential near the local minimum is $\mathcal{O}\left(m_{3 / 2}^{2} M_{\mathrm{P}}^{2}\right)$, this occurs at the critical temperature

$$
T_{\text {crit }} \sim \sqrt{m_{3 / 2} M_{\mathrm{P}}}
$$


where $m_{3 / 2}$ is the gravitino mass and $M_{\mathrm{P}}=2.4 \times 10^{18} \mathrm{GeV}$. An example of moduli destabilization by thermal corrections is shown in Fig. 3,

The critical temperature is defined by the appearance of a saddle point at some value $\Phi_{\text {crit }}$ :

$$
\begin{aligned}
& V_{\text {eff }}^{\prime}\left(\Phi_{\text {crit }}, T_{\text {crit }}\right)=0, \\
& V_{\text {eff }}^{\prime \prime}\left(\Phi_{\text {crit }}, T_{\text {crit }}\right)=0,
\end{aligned}
$$

where the prime indicates differentiation with respect to $\Phi$. Usually $V(\Phi)$ is a steep function, with exponential field dependence, while $V_{\text {th }}(\Phi)$ varies rather slowly. Thus, $V_{\text {th }}$ can be well approximated by a linear term in the region of interest and one obtains

$$
\begin{aligned}
V^{\prime \prime}\left(\Phi_{\text {crit }}\right) & =0 \\
T_{\text {crit }} & =\left(-\frac{V^{\prime}\left(\Phi_{\text {crit }}\right)}{v_{\text {th }}^{\prime}\left(\Phi_{\text {crit }}\right)}\right)^{1 / 4} .
\end{aligned}
$$

Note that $V^{\prime}\left(\Phi_{\text {crit }}\right)$ is the maximal slope of the supergravity potential.

It is important to realize that the moduli are not in thermal equilibrium. On the contrary, the moduli interaction rate $\Gamma_{\Phi} \sim T^{3} / M_{\mathrm{P}}^{2}$ is much smaller than the Hubble parameter $H \sim T^{2} / M_{\mathrm{P}}$. Hence, the moduli never reach thermal equilibrium. In particular, they do not attain a thermal mass ${ }^{1}$. Rather, they behave as classical backgrounds. This is analogous to the behaviour of the gravitational metric in a thermal bath where the thermal expectation value of the energy momentum tensor drives the cosmological expansion. On the other hand, gauge and/or matter fields are in thermal equilibrium and contribute to the effective potential through loops. In this way, the gauge plasma exerts a force on the classical backgrounds and drives the moduli.

Let us summarize the conditions under which Eq. (6) for the critical temperature in the radiation dominated phase applies:

(i) The modulus $\Phi$ is related to some coupling $g$.

(ii) The barrier separating a local minimum in $\Phi$ from the 'run-away' minimum is related to SUSY breaking.

(iii) Gauge and matter fields which couple with strength $g$ are in thermal equilibrium.

A few comments are in order. First, $g$ is not necessarily the gauge coupling. For instance, the Yukawa couplings of twisted states are functions of the $T$-moduli, $Y \sim$ $e^{-\alpha T}$. Then the diagram Fig. 1(c) generates an effective potential for these moduli, as long as the matter fields are in thermal equilibrium. Second, if $g$ is a gauge

\footnotetext{
${ }^{1}$ This is one of the main differences between our results and those of Ref. [9].
} 


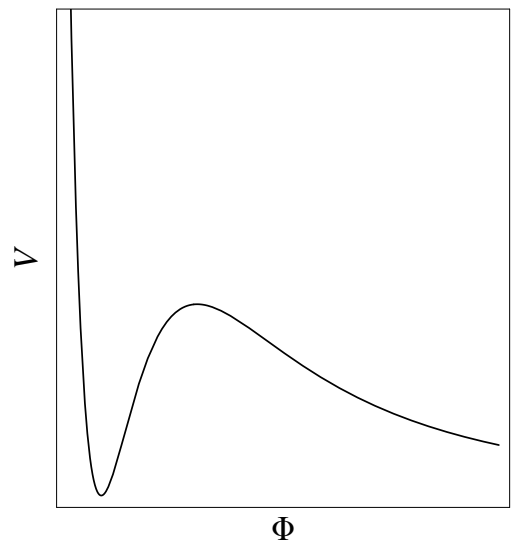

(a) $V$.

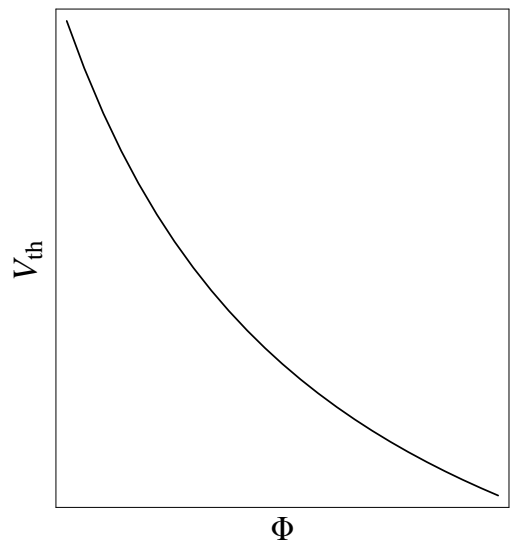

(b) $V_{\text {th }}$.

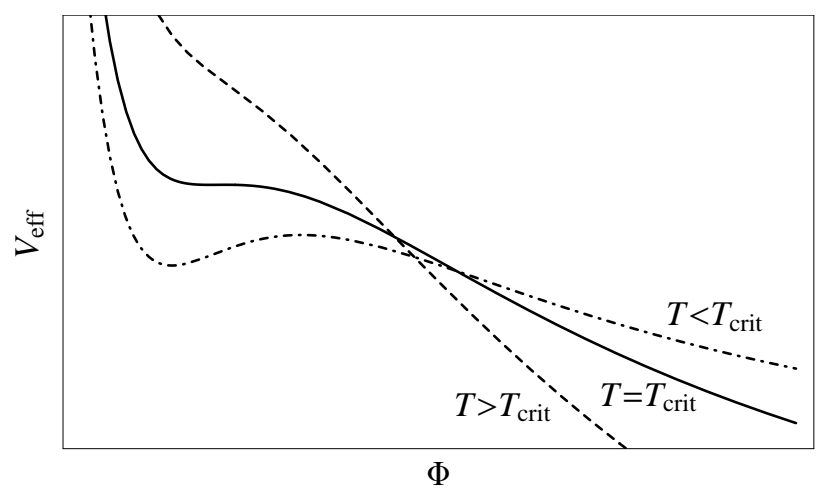

(c) $V_{\text {eff }}(\Phi, T)$.

Figure 3: Moduli destabilization by temperature corrections. (a) supergravity potential, (b) potential induced by thermal corrections, (c) evolution of the full potential with increasing temperature: $T<T_{\text {crit }}$ (dash-dotted curve), $T=T_{\text {crit }}$ (solid curve), and $T>T_{\text {crit }}$ (dashed curve).

coupling, $\Phi$ is not necessarily the dilaton. It can, for instance, be a volume modulus, as it happens in D-brane models. Third, Eq. (6) holds for positive, negative or zero cosmological constant, as long as condition (ii) is satisfied. 


\section{Critical temperature in the KKLT scenario}

Background fluxes induce a potential which can stabilize the dilaton and the complex structure moduli at appropriate values [2]. However, at least one Kähler modulus, corresponding to the overall volume, is not affected by the fluxes and remains undetermined. The volume can be stabilized by non-perturbative contributions to the superpotential such as D-brane instantons or gaugino condensation [1]. The result is a supersymmetric AdS vacuum.

In order to lift the negative cosmological constant of the AdS vacuum to a small positive value, extra contributions are added to the scalar potential. These can be due to the presence of $\overline{\mathrm{D} 3}$-branes, as in the original KKLT model or SUSY breaking D-terms [10]. A common feature of these modifications is that the new vacuum is separated from the 'run-away' vacuum [6] by a barrier whose height is given by the SUSY breaking scale (cf. Fig. 2).

In D-brane models, the gauge and matter fields of the standard model and the hidden sector can live on D3 or D7 branes [11]. At high temperature, thermalized gauge and matter fields of unbroken gauge groups on D7-branes will contribute to the scalar potential of the volume modulus. Depending on the D-brane model, the thermalized fields can belong to the standard model or to additional gauge groups for which no gaugino condensation occurs. This will lead to the existence of a maximal temperature beyond which the volume modulus is destabilized. In the following we calculate this critical temperature.

The superpotential and the Kähler potential for the volume modulus $\rho=\sigma+i \alpha$ are given by [1]

$$
\begin{aligned}
W & =W_{0}+A e^{-a \rho}, \\
K & =-3 \ln (2 \sigma) .
\end{aligned}
$$

Here $W_{0}$ is a constant induced by the fluxes, and $W-W_{0}$ represents a nonperturbative contribution to the superpotential due to $\mathrm{D}$-brane instantons or gaugino condensation. In the latter case the exponent is given by the $\beta$-function of the corresponding gauge group, $4 \pi a=3 /(2 \beta)$. The effective $4 \mathrm{D}$ Yang-Mills gauge coupling on the D7-branes is related to $\sigma$ as

$$
\sigma=\frac{4 \pi}{g^{2}}
$$

We can always choose the constant $W_{0}$ to be real and negative. $\alpha=\operatorname{Im}(\rho)$ is then stabilized at a value where the remaining potential for $\sigma=\operatorname{Re}(\rho) \operatorname{reads}$

$$
V_{0}=\frac{a A e^{-a \sigma}}{2 \sigma^{2}}\left[\frac{1}{3} a A \sigma e^{-a \sigma}+W_{0}+A e^{-a \sigma}\right],
$$

with $A$ real and positive. $V_{0}$ has an AdS minimum. This potential is amended by a supersymmetry breaking term,

$$
V=V_{0}+\frac{D_{n}}{\sigma^{n}}
$$


Here $n=3$ corresponds to the KKLT potential [1], which can be realized as a Fayet-Iliopoulos D-term term [10], whereas $n=2$ occurs for the explicitly SUSY breaking contribution of an $\overline{\mathrm{D} 3}$-brane [12].

For certain choices of the parameters, one can obtain $\mathrm{dS}$ vacua with a small cosmological constant. An example is $W_{0}=-10^{-4}, A=1, a=0.1, D_{3}=3 \times 10^{-9}$ $\left(D_{2}=2.6 \times 10^{-11}\right)$; for $n=3$ this is the KKLT potential. Both cases, $n=3$ and $n=2$ are shown in Fig. 4 Numerically, they are almost indistinguishable. The local minimum is at $\sigma_{\min } \simeq 115$, corresponding to the gauge coupling $g=0.3$.

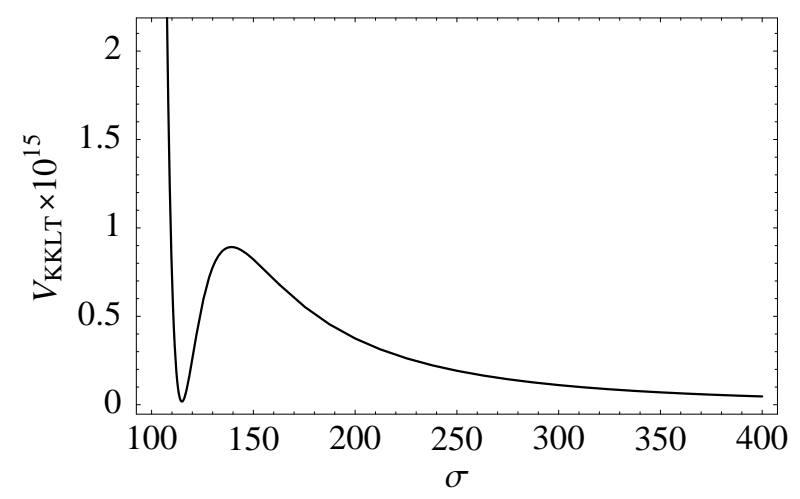

Figure 4: The KKLT potential.

To calculate the critical temperature, we need the first and the second derivative of the potential. For $\sigma \gg 1$ and $a \sigma \gg 1, V^{\prime}$ and $V^{\prime \prime}$ are well approximated by

$$
\begin{aligned}
V^{\prime} & \simeq-\frac{a^{3} A^{2}}{3 \sigma} e^{-2 a \sigma}-\frac{a^{2} A W_{0}}{2 \sigma^{2}} e^{-a \sigma}, \\
V^{\prime \prime} & \simeq \frac{2 a^{4} A^{2}}{3 \sigma} e^{-2 a \sigma}+\frac{a^{3} A W_{0}}{2 \sigma^{2}} e^{-a \sigma} .
\end{aligned}
$$

Note that for the derivatives $D_{n} / \sigma^{n}$ is negligible. Setting $V^{\prime \prime}=0$, which defines $\sigma_{\mathrm{cr}}$, one finds the maximal slope of the potential:

$$
V_{\max }^{\prime} \simeq \frac{a^{3} A^{2}}{3 \sigma_{\mathrm{cr}}} e^{-2 a \sigma_{\mathrm{cr}}} .
$$

It is straightforward to relate the maximal slope to the scale of supersymmetry breaking. Since the cosmological constant is negligibly small, the gravitino mass is given by

$$
\begin{aligned}
m_{3 / 2}^{2} & =\left.e^{K}|W|^{2}\right|_{\min }=-\frac{1}{3} V_{0}\left(\sigma_{\min }\right) \\
& \simeq \frac{a^{2} A^{2}}{18 \sigma_{\min }} e^{-2 a \sigma_{\min }} .
\end{aligned}
$$

For the KKLT parameters the gravitino mass is very large, $m_{3 / 2} \sim 10^{10} \mathrm{GeV}$. Note, that in the case of explicit SUSY breaking, $m_{3 / 2}$ is not the physical gravitino mass but just a parameter which controls the scale of SUSY breaking. Due to steepness 
of the potential, $\sigma_{\min }$ and $\sigma_{\text {cr }}$ are very close to each other. Hence, combining (15) and (16), one obtains

$$
V_{\max }^{\prime} \simeq 6 a m_{3 / 2}^{2} .
$$

From Eqs. (4) and (8) one now obtains for the critical temperature:

$$
T_{\text {crit }} \simeq c \sqrt{m_{3 / 2}},
$$

with $c \simeq\left|6 a / v_{\text {th }}^{\prime}\left(\sigma_{\mathrm{cr}}\right)\right|^{1 / 4}=\mathcal{O}(1)$. Note that this result holds both for $\delta V \propto 1 / \sigma^{3}$ and $\delta V \propto 1 / \sigma^{2}$. Numerically, for $m_{3 / 2} \sim 100 \mathrm{GeV}$, the maximal temperature is

$$
T_{\text {crit }} \sim 10^{10} \mathrm{GeV} .
$$

In the case of gaugino condensation one has $4 \pi a=3 /(2 \beta)$. Using $1 / g^{2}=4 \pi \sigma$, Eq. (18) reads explicitly

$$
T_{\text {crit }} \sim \sqrt{m_{3 / 2}}\left(\frac{2}{B}\right)^{1 / 4}\left(\frac{3}{\beta}\right)^{1 / 4}\left(\frac{1}{g^{2}}\right)^{3 / 8},
$$

where $B=\left(1 / T^{4}\right) \partial F / \partial g\left(\Phi_{\text {crit }}\right)[5]$. It is instructive to compare this result with racetrack models, where the Kähler potential is also logarithmic and the superpotential is a sum of two exponential terms. In this case the critical temperature is given by [5]

$$
T_{\text {crit }} \sim \sqrt{m_{3 / 2}}\left(\frac{2}{B}\right)^{1 / 4}\left(\frac{3}{\beta}\right)^{3 / 4}\left(\frac{1}{g^{2}}\right)^{7 / 8} .
$$

Clearly, both expressions are very similar. The different powers of $1 / \beta$ and $1 / g^{2}$ reflect the differences of the superpotential and Kähler potential in the two cases. For typical values, e.g. $1 / g^{2} \simeq 2$ and $\beta=0.1$, the critical temperature in racetrack models is larger by about a factor five.

\section{Discussion}

The maximal temperature derived above places a bound on the temperature in the radiation dominated phase of the early universe. In particular, it bounds the reheating temperature,

$$
T_{\text {reheat }}<T_{\text {crit }} \text {. }
$$

In addition, it places a bound on the maximal temperature in the preheating epoch [13],

$$
T_{\text {preheat }}=\left(T_{\text {reheat }}^{2} H_{\text {inf }} M_{\mathrm{P}}\right)^{1 / 4}<T_{\text {crit }},
$$

unless the inflaton coupling to $\Phi$ is strong enough to overcome the destabilizing thermal effects. This bound is usually more severe than (22), yet it is also less universal. 
We note that these bounds on the temperature of the early universe, unlike the gravitino bound, cannot be circumvented by late entropy production or other cosmological mechanisms. They are also independent of the 'overshoot problem' [14], that during the cosmological evolution a modulus with a steep potential may not settle in a shallow minimum, but rather roll over the barrier to infinity. This problem can be solved by tuning the initial conditions or by implementing a mechanism to slow down the modulus (cf. [15] and references therein). On the contrary, the constraint $T<T_{\text {crit }}$ is unavoidable since there is no local minimum for $T>T_{\text {crit }}$ and the modulus inevitably runs away to infinity.

While finalizing this work we received a related paper by Kallosh and Linde [16] which addresses moduli destabilization during inflation in KKLT models. The authors also present a model where, at special points in parameter space, the size of the barrier separating the physical vacuum from the unphysical one is unrelated to the gravitino mass. However, for generic parameters, the barrier is related to the scale of supersymmetry breaking and the analysis of the present paper applies.

Acknowledgements. We would like to thank R. Brustein for correspondence, and J. Louis and H.-P. Nilles for discussions. One of us (M.R.) would like to thank the Aspen Center for Physics for support. This work was partially supported by the EU 6th Framework Program MRTN-CT-2004-503369 "Quest for Unification" and MRTN-CT-2004-005104 "ForcesUniverse".

\section{References}

[1] S. Kachru, R. Kallosh, A. Linde and S. P. Trivedi, Phys. Rev. D 68 (2003) 046005 .

[2] S. B. Giddings, S. Kachru and J. Polchinski, Phys. Rev. D 66 (2002) 106006.

[3] J. P. Derendinger, L. E. Ibáñez and H. P. Nilles, Phys. Lett. B 155 (1985) 65; M. Dine, R. Rohm, N. Seiberg and E. Witten, Phys. Lett. B 156 (1985) 55.

[4] W. Buchmüller, K. Hamaguchi and M. Ratz, Phys. Lett. B 574 (2003) 156.

[5] W. Buchmüller, K. Hamaguchi, O. Lebedev and M. Ratz, Nucl. Phys. B 699 (2004) 292.

[6] M. Dine and N. Seiberg, Phys. Lett. B 162 (1985) 299.

[7] I. Navarro and J. Santiago, JCAP 0409 (2004) 005.

[8] J. I. Kapusta, "Finite Temperature Field Theory," Cambridge, 1989.

[9] P. Binetruy and M. K. Gaillard, Phys. Rev. D 34 (1986) 3069; K. A. Olive and M. A. Srednicki, Phys. Lett. B 148 (1984) 437.

[10] C. P. Burgess, R. Kallosh and F. Quevedo, JHEP 0310 (2003) 056. 
[11] P. G. Camara, L. E. Ibáñez and A. M. Uranga, Nucl. Phys. B 689 (2004) 195; hep-th/0408036 M. Graña, T. W. Grimm, H. Jockers and J. Louis, Nucl. Phys. B 690 (2004) 21; F. Marchesano and G. Shiu, hep-th/0408059, hep-th/0409132 H. Jockers and J. Louis, hep-th/0409098 D. Lüst, S. Reffert and S. Stieberger, hep-th/0410074.

[12] S. Kachru, R. Kallosh, A. Linde, J. Maldacena, L. McAllister and S. P. Trivedi, JCAP 0310 (2003) 013.

[13] E. Kolb, M. Turner, The Early Universe, Addison-Wesley, Redwood City, CA 1990.

[14] R. Brustein and P. J. Steinhardt, Phys. Lett. B 302 (1993) 196.

[15] R. Brustein, S. P. de Alwis and P. Martens, hep-th/0408160 N. Kaloper, J. Rahmfeld and L. Sorbo, hep-th/0409226.

[16] R. Kallosh and A. Linde, hep-th/0411011. 